\title{
Identification of Der f 23 as a new major allergen of Dermatophagoides farinae
}

\author{
YONGSHEN HE ${ }^{1}$, CHUANRAN DOU $^{2}$, YIMING SU ${ }^{3}$, JIALIN CHEN ${ }^{1}$, \\ ZHEN ZHANG $^{1}$, ZHENFU ZHAO ${ }^{1}$, JIAJIE CHEN ${ }^{1}$ and KUNMEI JI ${ }^{1}$ \\ ${ }^{1}$ Department of Biochemistry and Molecular Biology, Health Science Center of Shenzhen University, Shenzhen, \\ Guangdong 518060; ${ }^{2}$ Shenzhen Vanke Meisha Academy, Shenzhen, Guangdong 518000; \\ ${ }^{3}$ The Affiliated International School of Shenzhen University, Shenzhen, Guangdong 518054, P.R. China
}

Received November 7, 2018; Accepted April 24, 2019

DOI: $10.3892 / \mathrm{mmr} .2019 .10305$

\begin{abstract}
House dust mites (HDM) are common allergen sources worldwide. At present, 32 of the 37 internationally recognized HDM allergen groups have been identified in Dermatophagoides farinae. The present study study describes the identification of the first known $D$. farinae Group 23 allergen (Der f 23). Recombinant Der f 23 protein (rDer f 23) was cloned, expressed and purified. The open reading frame of rDer f 23 was 525 base pairs and encoded a 174-amino acid protein (GenBank accession no., KU166910.1). ELISAs indicated that 72/129 HDM allergic serum samples (55.8\%) had specific immunoglobulin E (sIgE) binding activity to rDer f 23. Additionally, 3/10 patients with HDM allergies (30\%) exhibited positive skin prick test reactions to rDer $\mathrm{f} 23$. IgE western blot analysis data suggested that only 4/11 HDM allergic sera had a positive sIgE binding result. Sequence homology analysis revealed an extra P2 region (Ser56-Thr117) in Der f 23 that was not present in the D. pteronyssinus homolog, which may affect sIgE binding. Der f $23 \Delta \mathrm{P} 2$ demonstrated binding with HDM allergic sera, whereas the $\mathrm{P} 2$ peptide alone did not. The sIgE binding ability of Der f $23 \Delta \mathrm{P} 2$ (Der f 23 with a truncated $\mathrm{P} 2$ region) was more marked compared with that of Der $\mathrm{f} 23$ in an IgE ELISA. These data indicate that $\mathrm{P} 2$ region in Der f 23 attenuates IgE binding ability. In conclusion, the
\end{abstract}

Correspondence to: Professor Kunmei Ji or Dr Jiajie Chen, Department of Biochemistry and Molecular Biology, Health Science Center of Shenzhen University, 1066 Xueyuan Road, Nanshan, Shenzhen, Guangdong 518060, P.R. China

E-mail: jkm@szu.edu.cn

E-mail: chenjj@szu.edu.cn

Abbreviations: HDM, house dust mite; Der f, Dermatophagoides farinae; Der p, Dermatophagoides pteronyssinus; Der f 23, Group 23 allergen of Dermatophagoides farinae; Der p 23, Group 23 allergen of Dermatophagoides pteronyssinus; IPTG, isopropyl- $\beta$-D-thiogalac topyranoside; HRP, horseradish peroxidase

Key words: Dermatophagoides farinae, Der f 23, house dust mite, novel allergen results of the present study indicate that Der $\mathrm{f} 23$ is a major HDM allergen with predominantly conformational sIgE binding epitopes. The allergenic identification of Der f 23 and its inclusion in World Health Organization/International Union of Immunological Societies database contributes to the theoretical basis underlying the diagnosis and treatment of HDM allergic diseases.

\section{Introduction}

House dust mites (HDMs) are common indoor sources of allergens $(1,2)$. The primary species of HDM are Dermatophagoides pteronyssinus, D. farinae, Euroglyphus maynei and Blomia tropicalis $(3,4)$, with the former two being ubiquitous in home dust samples in temperate and tropical regions $(5,6)$. HDM allergens constitute a major cause of allergic diseases $(7,8)$, with one-half of allergy sufferers exhibiting an allergic reaction to HDM allergens $(9,10)$.

Clinicians use HDM allergen proteins to diagnose and treat HDM allergies (11,12). The HDM antigens used clinically are obtained from a crude HDM extract $(13,14)$. As these crude extracts are a mixture of a small portion of allergens and a number of unrelated impurities, their effects are highly varied, including adverse side effects in certain patients (15). Of the 39 HDM allergen groups recognized in the World Health Organization and International Union of Immunological Societies (WHO/IUIS) allergen database, 33 include identified $D$. farinae allergens and only 23 include $D$. pteronyssinus allergens (16). The identification of HDM allergens, particularly the detection and naming of novel HDM allergens, has direct significance for the diagnosis and treatment of HDM-induced allergic diseases.

Previous studies have suggested that the major HDM allergens belong to Group 1 (17,18), Group 2 (19,20), Group $23(21,22)$ and Group 24 (23). A Group 23 allergen from D. pteronyssinus (Der p 23) was identified to be a major allergen present in HDM feces in dust $(24,25)$. Der p 23 has been demonstrated to react with specific immunoglobulin Es (sIgEs) from $74 \%$ of patients with D. pteronyssinus allergies, which is smaller proportion compared with the positive reaction rates of the two previously recognized major HDM allergens, namely Der p 1 and Der p 2 (21). Der p 23 is a 
small, globular protein stabilized by 2 disulfide bonds that is structurally similar to other allergens, including Blot 12, in that it contains carbohydrate-binding domains that bind chitin (26).

To the best of our knowledge, the Group 23 allergen in D. farinae (Der f 23) had not been identified and characterized prior to the present study. The aims of this study were firstly to confirm the existence of Der f 23, and secondly to characterize the sIgE binding activity of Der f 23, if such an allergen was able to be isolated. In vitro IgE binding was determined by IgE western blot analysis, dot blot assays and ELISAs; in vivo reactivity was assayed with a skin prick test (SPT). The identification of a novel Der $\mathrm{f} 23$ allergen would be clinically useful for the diagnosis and treatment of HDM-induced allergic diseases.

\section{Materials and methods}

Materials. A D. farinae cDNA library preserved by the School of Medicine, Shenzhen University (Shenzhen, China) was employed. E. coli BL21 (DE3) plysS cells were purchased from Merck KGaA, and the pMD 19-T vector was purchased from Takara Biotechnology Co., Ltd. The pET-His and pET-His-DsbA vectors were purchased from Wuhan Miaoling Bioscience \& Technology Co., Ltd. The DNA sequence encoding the Der f $23 \Delta$ P2 protein (P2) was synthesized by Nanjing GenScript Biotech Corp. The Primer STAR HS DNA polymerase was purchased from Takara Biotechnology Co., Ltd. The lysozyme was purchased from Sangon Biotech Co., Ltd. The nitrocellulose and polyvinylidene fluoride (PVDF) membranes were purchased from Merck KGaA.

Serum samples from HDM-sensitive individuals, referred to as HDM allergic sera, and non-allergic individuals were provided by the First Affiliated Hospital of Guangzhou Medical College. Sera from non-allergic individuals were used for control group. A cohort of 129 subjects (65 males and 64 females; age range, 18-55 years) were enrolled. Samples from 31 non-allergic individuals were used as negative controls. The HDM-specific IgEs within the sera samples were assayed using an ImmunoCAP system (Thermo Fisher Scientific, Inc.). Ethical approval was obtained from the First Affiliated Hospital of Guangzhou Medical College (approval no. 2012-51). All procedures involving human participants were in accordance with the ethical standards of the committee of the First Affiliated Hospital of Guangzhou Medical College. All participants voluntarily agreed to participate, and all provided written informed consent.

\section{Methods}

cDNA cloning, protein expression and purification. Der f 23 open reading frame (ORF) cDNA was amplified by polymerase chain reaction (PCR) using the Primer STAR HS DNA polymerase with the $D$. farinae cDNA library. The primers used were: Forward, 5'-ATGAAATTCAACATA ACTAT CGC-3'; and reverse, 5'-TTATGTACATGTTAATTCTTTT TCA-3'. The PCR thermocycling conditions were $94^{\circ} \mathrm{C}$ for $30 \mathrm{sec}, 55^{\circ} \mathrm{C}$ for $30 \mathrm{sec}$ and $72^{\circ} \mathrm{C}$ for $50 \mathrm{sec}$, for 30 cycles. The PCR product, confirmed by DNA sequencing (GenScript Biotech Corporation), was subcloned into a pET-His vector, and then transformed into Escherichia coli (E. coli) BL21
(DE3) plysS. E. coli were grown overnight in Luria-Bertani medium (Thermo Fisher Scientific, Inc.) containing $100 \mathrm{mg} / \mathrm{l}$ ampicillin at $37^{\circ} \mathrm{C}$, and were induced by adding isopropyl- $\beta$ D-thiogalactopyranoside (IPTG) to a final concentration of $0.5 \mathrm{mM}$. Following cultivation for additional $3 \mathrm{~h}$ at $37^{\circ} \mathrm{C}$, $E$. coli cells were harvested by centrifugation at $9,600 \mathrm{x} \mathrm{g}$ for $5 \mathrm{~min}$ at $4^{\circ} \mathrm{C}$. Following mixing with the protein extraction buffer (20 mM PB, $150 \mathrm{mM} \mathrm{NaCl}$ and $1 \mathrm{mg} / \mathrm{ml}$ lysozyme), the harvested cells were sonicated for $3 \mathrm{sec}$ with 5 -sec intervals in an ice bath for a total of $20 \mathrm{~min}$, and then centrifuged again at $9,600 \mathrm{~g}$ for $20 \mathrm{~min}$ at $4^{\circ} \mathrm{C}$. The recombinant protein in the soluble portion was purified with a Ni-NTA column (cat. no. 17040303; GE Healthcare) and gel filtration (HiLoad Superdex 16/600; cat. no. 28-9893-33; GE Healthcare).

$\operatorname{IgE}$ western blot analysis and IgE dot blot assays. The protein concentration was determined by Bradford assay (Sangon Biotech Co., Ltd). The protein samples were diluted to $1 \mathrm{mg} / \mathrm{ml}$ and subjected to electro-transfer onto the PVDF membrane through $12 \%$ SDS-PAGE (20 $\mu \mathrm{g} / \mathrm{lane}$ ) for the IgE western blot analysis. Subsequently, the membranes were blocked overnight at $4^{\circ} \mathrm{C}$ with $5 \%$ skim milk in TBS $+0.05 \%$ Tween-20 (TBST). Then, the membranes were incubated with allergic sera (diluted in 1:10 vol/vol in PBS buffer) for $2 \mathrm{~h}$ at $37^{\circ} \mathrm{C}$. Following washing, the membranes were incubated with a horseradish peroxidase (HRP)-conjugated anti-human IgE antibody for $2 \mathrm{~h}$ at $37^{\circ} \mathrm{C}(1: 2,000$; cat. no. 9160-05; SouthernBiotech). The proteins were visualized for $10 \mathrm{~min}$ using 3,3'-diaminobenzidine (1:10; cat. no. 34002; Thermo Fisher Scientific, Inc.) at room temperature.

The $\operatorname{IgE}$ dot blot assay was performed as previously described (23). The protein samples were diluted to $1 \mathrm{mg} / \mathrm{ml}$, and $2 \mu \mathrm{l}$ samples were added to the nitrocellulose membrane for the $\operatorname{IgE}$ dot blot assay. Subsequently, the membranes were dried and blocked overnight at $4^{\circ} \mathrm{C}$ with $5 \%$ skim milk in TBST. Then, the membranes were incubated with allergic sera (diluted in 1:10 vol/vol in PBS buffer) for $2 \mathrm{~h}$ at $37^{\circ} \mathrm{C}$. Following washing, the membranes were incubated with the HRP-conjugated anti-human IgE antibody for $2 \mathrm{~h}$ at $37^{\circ} \mathrm{C}$ (1:2,000; cat. no. 9160-05; SouthernBiotech). The proteins were visualized for 10 min using 3,3'-diaminobenzidine (1:10; cat. no. 34002; Thermo Fisher Scientific, Inc.).

IgE ELISA. The IgE ELISA assay developed in the present study was performed as previously described (23). The isolated protein was added to the ELISA plate at $100 \mathrm{ng} /$ well and coated overnight at $4^{\circ} \mathrm{C}$. The coated plate was blocked with $5 \%$ skim milk in PBS $+0.1 \%$ Tween-20 (PBST) for $2 \mathrm{~h}$ at $37^{\circ} \mathrm{C}$. Then, the plate was incubated with allergic sera (diluted 1:10 vol/vol in $1 \%$ skim milk-PBST) for $2 \mathrm{~h}$ at $37^{\circ} \mathrm{C}$. IgEs were detected with the HRP-conjugated mouse anti-human IgE antibody (1:2,000 in 1\% skim milk-PBST; cat. no. 9160-05; SouthernBiotech). Binding reactions were visualized by adding tetramethyl benzidine substrate, and absorbance at $450 \mathrm{~nm}$ was measured by a microplate reader (Bio-Rad Laboratories, Inc.).

SPTs. SPTs were performed with rDer f 23 protein $(10 \mathrm{mg} / \mathrm{ml})$ and standardized D. farinae extract (ALK Abelló A/S) in 10 patients with allergic rhinitis and/or asthma and 10 healthy controls (Table I) (23). The response was observed 15 min after 
Table I. Skin reactivity to $\operatorname{Der} f$ extracts and $\mathrm{rDer} f 23$ protein $(10 \mu \mathrm{g} / \mathrm{ml})$.

\begin{tabular}{|c|c|c|c|c|}
\hline \multirow[b]{2}{*}{ Patient no. } & \multirow[b]{2}{*}{ Sex/age } & \multicolumn{3}{|c|}{ Skin prick tests ${ }^{\mathrm{a}}$} \\
\hline & & Clinical history & $\operatorname{Der} f$ extracts & rDer f 23 \\
\hline 1 & Female/39 & AR & $3+$ & $2+$ \\
\hline 2 & Female/38 & $\mathrm{AR}+\mathrm{BA}$ & $3+$ & - \\
\hline 3 & Male/25 & AR & $3+$ & - \\
\hline 4 & Female/40 & AR & $3+$ & - \\
\hline 5 & Male/52 & AR & $4+$ & $2+$ \\
\hline 6 & Female/28 & BA & $3+$ & - \\
\hline 7 & Male/24 & AR & $4+$ & $2+$ \\
\hline 8 & Female/23 & AR & $3+$ & - \\
\hline 9 & Male/38 & $\mathrm{BA}$ & $3+$ & - \\
\hline 10 & Male/44 & $\mathrm{AR}+\mathrm{BA}$ & $3+$ & - \\
\hline
\end{tabular}

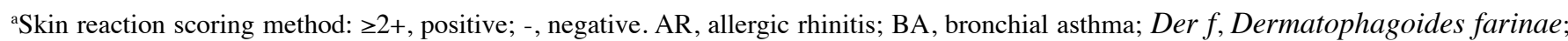
rDer f 23, recombinant Dermatophagoides farinae 23 protein.

pricking. The result was considered positive when the prick spot developed a wheal with a surrounding fleck; no visible response was considered a negative result.

Sequence homology. The nucleotide sequences of Der f 23 (GenBank: KU166910.1) and Der p 23 (GenBank: KP895831) were imported into DNAMAN 8 software (version 8.0; Lynnon Biosoft) for alignment. The sequences were saved in FASTA format for subsequent analysis.

Expression and purification of Der $f 23 \triangle P 2$ and $P 2$ proteins. Der $\mathrm{f} 23 \Delta \mathrm{P} 2$ and $\mathrm{P} 2$ region gene sequences were synthesized by Nanjing GenScript Biotech Corp., and each was subcloned into the pET-His-DsbA vector. The recombinant proteins were expressed and purified as aforementioned. Specifically, the Der f $23 \Delta \mathrm{P} 2$ protein was expressed in inclusion bodies and was dissolved in $6 \mathrm{M}$ urea with $25 \mathrm{mM} \beta$-mercaptoethanol. For the renaturation process, the Der f $23 \Delta \mathrm{P} 2$ protein was dialyzed with PBS at $4^{\circ} \mathrm{C}$ for $24 \mathrm{~h}$ using a dialysis membrane (cat. no. F132550; Sangon Biotech Co., Ltd.). Additionally, soluble recombinant DsbA-P2 protein was obtained. The purified protein Der f $23 \Delta \mathrm{P} 2$ and DsbA-P2 were subjected to in vitro $\mathrm{IgE}$ binding assays.

Statistical analysis. Quantitative data are presented as means \pm standard error of the mean. Analyses were performed using GraphPad Prism 7 software (GraphPad Software, Inc.). Differences between the allergic and control groups were determined by one-way ANOVA followed by Dunnett's post-hoc test for multiple comparisons. $\mathrm{P}<0.05$ was considered to indicate a statistically significant difference.

\section{Results}

Amino acid sequence homology between Der $f 23$ and Der $p$ 23 . The Der $\mathrm{f} 23 \mathrm{ORF}$ sequence was cloned using the $D$. farinae cDNA library as a template, and specific Der f 23 sequence primers were designed based on the $D$. farinae genome in the National Center for Biotechnology Information (NCBI) database (Genome ID: 9138). DNA sequencing confirmed the sequence of the ORF cDNA of Der f 23 to be 525 base pairs, and the sequence was registered in the NCBI library (GenBank: KU166910.1). A homology comparison conducted in DNAMAN 8 software indicated that Der f 23 has 173 amino acids, whereas Der p 23 has 97 amino acids, with the additional amino acids constituting 2 extra regions, namely P1 (Phe26-Pro38) and P2 (Ser56-Thr117), as demonstrated in Fig. 1.

Cloning, expression and purification of Der $f 23$. To obtain the Der f 23 protein, a prokaryotic pET-His vector was constructed and transformed into $E$. coli for expression and purification. The Der f 23 ORF DNA sequence obtained by PCR amplification with specific primers, as demonstrated in Fig. 2A, was cloned into a pET-His vector. The pET-His-Der f 23 vector was identified by double digestion with $B a m \mathrm{H} \mathrm{I}$ and HindIII (Fig. 2B). Subsequently, the pET-His-Der f 23 vector was transformed into $E$. coli BL21 (DE3) plysS cells for expression and purification. SDS-PAGE analysis demonstrated that Der f $23(\sim 30 \mathrm{kDa})$ was successfully expressed in the supernatant following IPTG induction and purification with Ni-NTA-resin (Fig. 2C and D).

IgE binding of Der $f 23$. The IgE ELISA indicated that HDM allergic sera from 72/129 patients with HDM allergies (55.8\%) exhibited sIgE binding activity with Der f 23 (Fig. 3A). None of the serum samples from 31 non-allergic individuals were reactive (Fig. 3A). Data from the IgE dot blot assays suggested that Der $\mathrm{f} 23$ protein exhibited a good sIgE binding capacity for HDM allergic sera, but not for sera from non-allergic individuals. Der f $1 / 2$ fusion protein was used as positive control (Fig. 3B). In the IgE western blot analysis, only 4/11 selected IgE ELISA reactive sera exhibited sIgE reactivity to $r$ Der $\mathrm{f} 23$ (Fig. 3D), suggesting that sIgE binding epitopes in Der f 23 may be conformation-dependent. As demonstrated in Table I, $3 / 10(30 \%)$ of patients with HDM allergies exhibited a positive in vivo reaction to Der f 23 in the SPTs. Based on these 


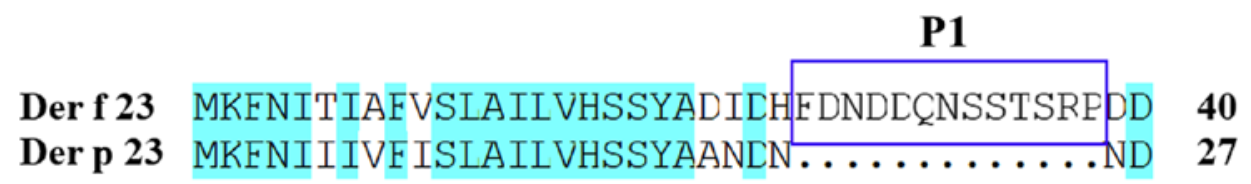

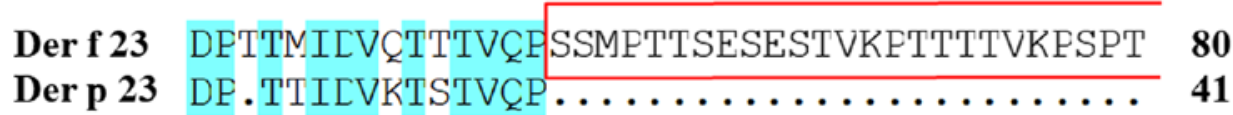

P2

Der f23 TVKPTTTTVKLTTTTVKPSPTTVKPTTTTVKPSPTTTTTT $\mathbf{1 2 0}$

Derp $23 \ldots \ldots \ldots \ldots \ldots \ldots \ldots \ldots \ldots \ldots \ldots \ldots \ldots \ldots \ldots \ldots \ldots \ldots$

$\begin{array}{lll}\text { Der f 23 } & T T E Q F E E E E C F T R F G Y F A D F K C C K F Y I C S N W E A I H K S C & \mathbf{1 6 0} \\ \text { Der p 23 } & T T K Q F D E E F E C F T R F G F A D F K C F H K F I C S N W E A V H K D C & \mathbf{8 4}\end{array}$

Der f 23 PGNTRWNEKELTC

173

Der p 23 PGNTRWNELEETC

Figure 1. Amino acid sequence homology between Der f 23 and Der p 23. The common amino acids are shaded in blue. The different regions, P1 (Phe26-Pro38) and P2 (Ser56-Thr117), are highlighted in blue and red boxes, respectively. Der f, Dermatophagoides farinae; Der p, Dermatophagoides pteronyssinus.

results, the WHO/IUIS Allergen Nomenclature subcommittee published this allergenic HDM protein as Der f 23 (16).

Involvement of $P 2$ region (Ser56-Thrl17) in sIgE binding. The $\mathrm{P} 2$ region peptide and Der f $23 \Delta \mathrm{P} 2$ protein were expressed in E. coli. DsbA-Der f $23 \Delta \mathrm{P} 2$ was observed in the form of inclusion bodies with a molecular weight of $\sim 38 \mathrm{kDa}$ and DsbA-P2 was soluble with a molecular weight of $\sim 35 \mathrm{kDa}$ (Fig. 4A). The IgE ELISA demonstrated that $r$ Der $\mathrm{f} 23$ and DsbA-Der $\mathrm{f} 23 \Delta \mathrm{P} 2$ exhibited significant binding activity, with all 8 HDM allergic sera tested, whereas $\mathrm{P} 2$ exhibited no IgE binding reactivity with any of the sera (Fig. 4B). The DsbA protein served as a negative control for sIgE binding activity (data not shown). Notably, within the same positive sera, the optical density value of DsbA-Der $f$ $23 \Delta \mathrm{P} 2$ was increased compared with that of $r$ Der $\mathrm{f} 23(\mathrm{P}<0.05)$. Similarly, the dot blot assay results confirmed that DsbA-Der f $23 \Delta \mathrm{P} 2$ exhibited significant sIgE binding reactivity, while the $\mathrm{P} 2$ peptide did not. The color reaction was more marked for DsbA-Der f $23 \Delta \mathrm{P} 2$ compared with the Der f 23. These data indicated that the $\mathrm{P} 2$ region of Der $\mathrm{f} 23$ may affect the Der $\mathrm{f} 23$ protein structure and thereby attenuate the $\operatorname{IgE}$ binding ability of Der $\mathrm{f} 23$ in sera.

\section{Discussion}

The present study cloned, expressed and purified rDer $\mathrm{f} 23$ protein using an E. coli expression system. The resultant recombinant protein exhibited SIgE binding activity in vitro and in vivo, and was therefore included as Der $\mathrm{f} 23$ in the WHO/IUIS allergen database (16). The present study identified that, in comparison with Der p 23, Der f 23 contained an extra P2 region that affected its IgE binding ability. In the IgE ELISAs, $72 / 129$ positive sera $(55.81 \%)$ from patients with HDM allergies exhibited marked sIgE binding activity to $\mathrm{rDer}$ f 23 protein. These data confirmed that Der $\mathrm{f} 23$ is a major allergen, consistent with previous results for Der $\mathrm{p}$ $23(21)$.

The allergen components of the $2 \mathrm{HDM}$ species $D$. farinae and $D$. pteronyssinus are highly homologous, encompassing 39 HDM allergen groups (16). Indeed, the Der p 23 allergen identified in the present study had $71 \%$ amino acid sequence homology with Der f 23. Additionally, it was identified that Der p 23 had 2 extra regions not present in Der f 23, namely a P1 region (Phe26-Pro38) and a P2 region (Ser56-Thr117). These sequence differences may be the underlying cause of a differential allergenic effect between Der f 23 and Der p 23 proteins, and therefore an allergic distinction between the 2 HDM species of D. farinae and D. pteronyssinus. In light of recent evidence demonstrating the existence of multiple Der f 23 isoforms (27), we hypothesized that the sequence difference between Der f 23 and Der p 23 may be due to the insertion of an intron in the gene that encodes Der $\mathrm{f} 23$.

The IgE binding ability of HDM allergens is based primarily on B-cell epitopes of allergens, which may occur in conformational or linear form. Conformational epitopes are more favorable for IgE binding compared with linear epitopes (28). Conformational changes in IgEs contribute to a decrease in their dissociation rates from high-affinity IgE receptors (29). B-cell epitopes are primarily conformational, including some that are discontinuous, in which polypeptide chain folding draws distant amino acid segments in the primary structure into close proximity on the surface of the molecule $(30,31)$. These regions are able to form complementarity-determining regions recognized by 

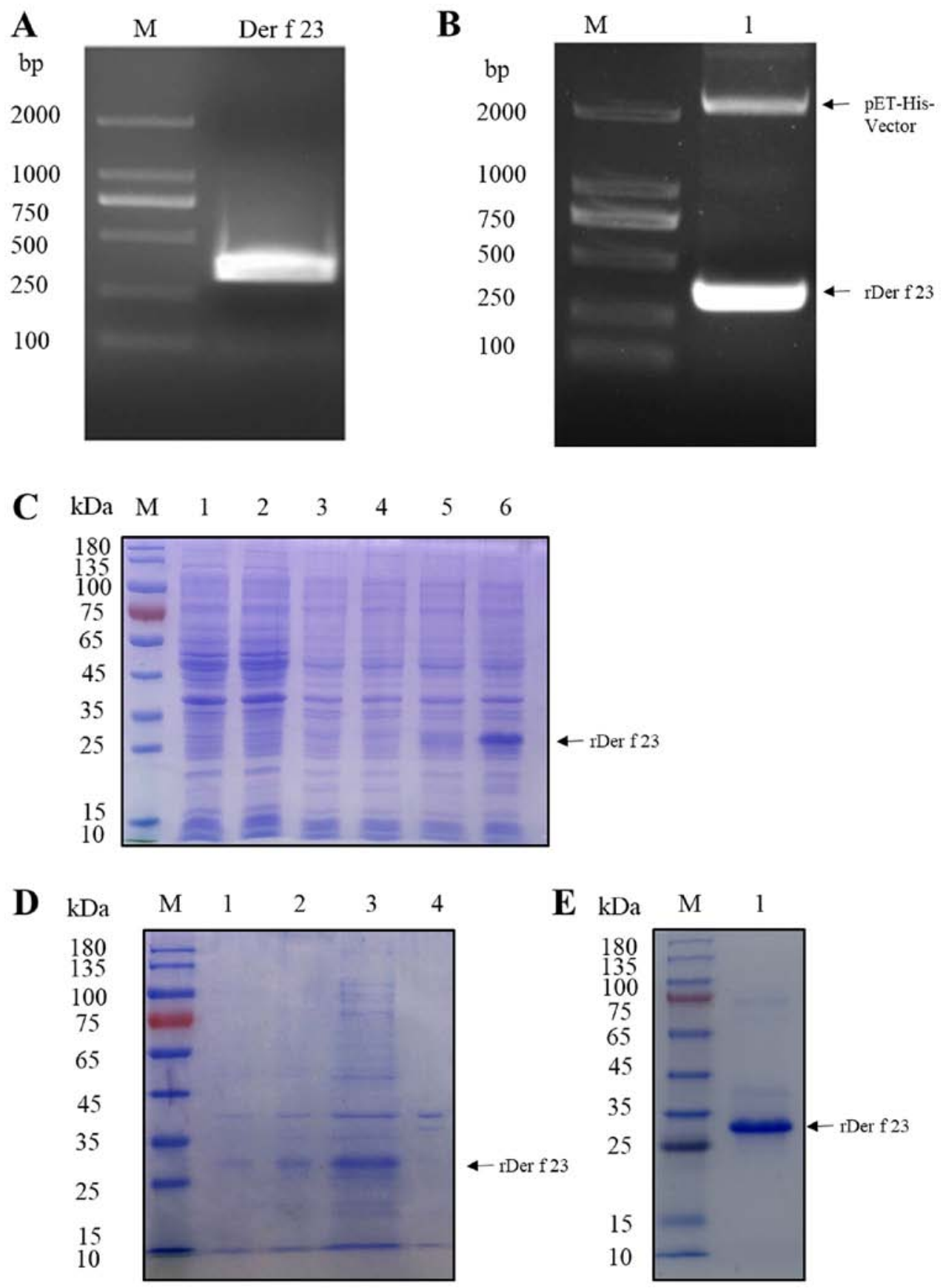

Figure 2. Cloning, expression and purification of rDer $\mathrm{f} 23$. (A) Cloning of Der $\mathrm{f} 23 \mathrm{cDNA}$ by polymerase chain reaction. Lane M, DL2000 marker; lane 1, Der f 23 cDNA PCR product. (B) Analysis of expression vector pET-His-Der $\mathrm{f} 23$ by double enzyme digestion. Lane M, DL2000 Marker; lane 1, pET-His-Der $\mathrm{f}$ 23 vector digested by BamH I/HindIII. (C) Analysis of purification of pET-His-Der f 23 by SDS-PAGE. Lane M, protein marker; lane 1, cell lysate of E. coli without induction; lane 2, cell lysate of E. coli following IPTG induction; lane 3, E. coli transformed with pET-His-vector without induction; lane 4m, cell lysate of pET-His-vector following IPTG induction; lane 5, E. coli transformed with pET-Der f 23 prior to IPTG induction; lane 6, E. coli transformed with pET-Der $\mathrm{f} 23$ following IPTG induction. (D) Analysis of expression of $\mathrm{rDer} \mathrm{f} 23$ in an E. coli system. Lane M, protein marker; lane 1, E. coli transformed with pET-Der $\mathrm{f} 23$ prior to IPTG induction; lane 2, E. coli transformed with pET-Der $\mathrm{f} 23$ following IPTG induction; lane 3, supernatant from $E$. coli transformed with pET-His-Der f 23 plasmid following IPTG induction, subsequent to ultrasonication; lane 4, sediment from E. coli samples transformed with pET-His-Der $\mathrm{f} 23$ plasmid following IPTG induction, subsequent to ultrasonication. (E) SDS-PAGE of purified $\mathrm{rDer} \mathrm{f}$ 23. Lane M, protein marker; lane 1, purified rDer $\mathrm{f} 23$. E. coli, Escherichia coli; rDer f 23, recombinant Dermatophagoides farinae 23 protein; IPTG, isopropyl- $\beta$-D-thiogalactopyranoside.

antibodies (32). The results from the present study, that only 4/11 sera with a positive IgE ELISA result also exhibited a positive $\operatorname{IgE}$ western blot analysis result suggests that the $\operatorname{IgE}$ epitope of Der f 23 may be predominantly a conformational epitope, consistent with the data from Szalai et al (33), which suggested that the B-cell epitopes of Der $\mathrm{p} 1$ and Der $\mathrm{p} 2$ are conformational.
Despite their high degrees of homology, different HDM allergen components within the same group have differing IgE binding abilities determined by their amino acid sequences and protein structures. For example, although Der p 1 and Der $\mathrm{f} 1$ share an extensive sequence identity, differences identified in their crystal structures may explain the differences in human IgE antibody responses to these 
A

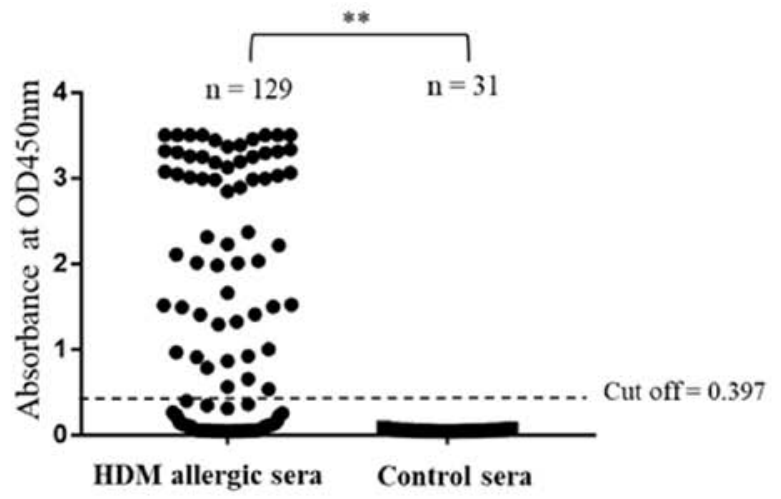

B

Individual HDM allergic sera

\begin{tabular}{|c|c|c|c|c|c|c|c|c|c|c|c|}
\hline & 1 & 2 & 3 & 4 & 5 & 6 & 7 & 8 & 9 & 10 & 11 \\
\hline rDer f 23 & & & & C & & & & & & & \\
\hline rDer f $1 / 2$ & e & 0 & (- & 0 & 0 & 0 & 0 & ? & 0 & 0 & O \\
\hline
\end{tabular}
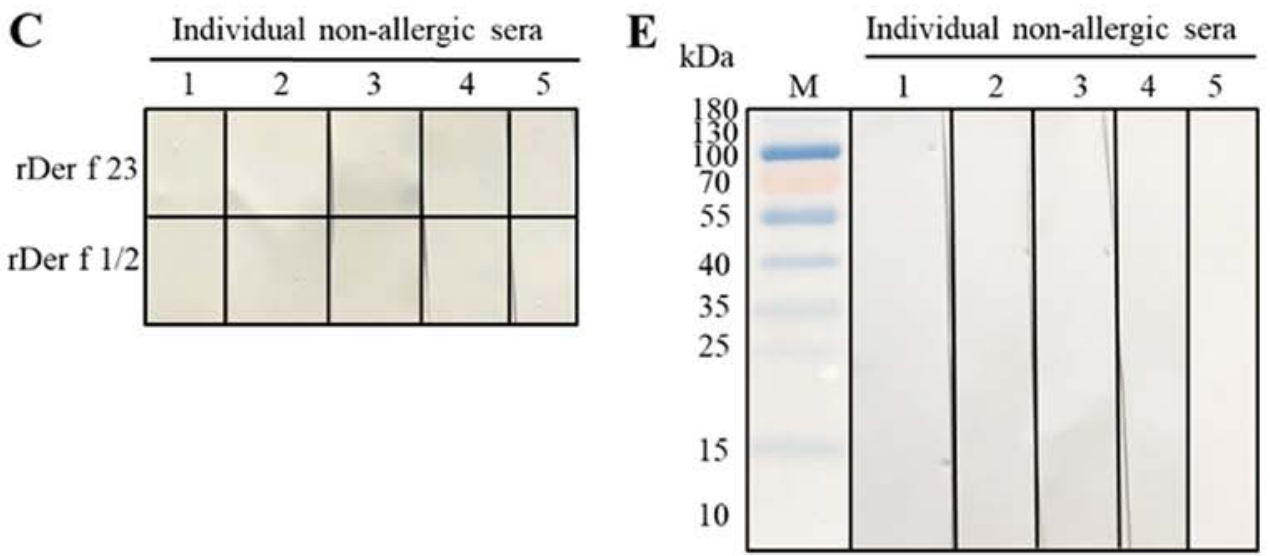

D Individual HDM allergic sera

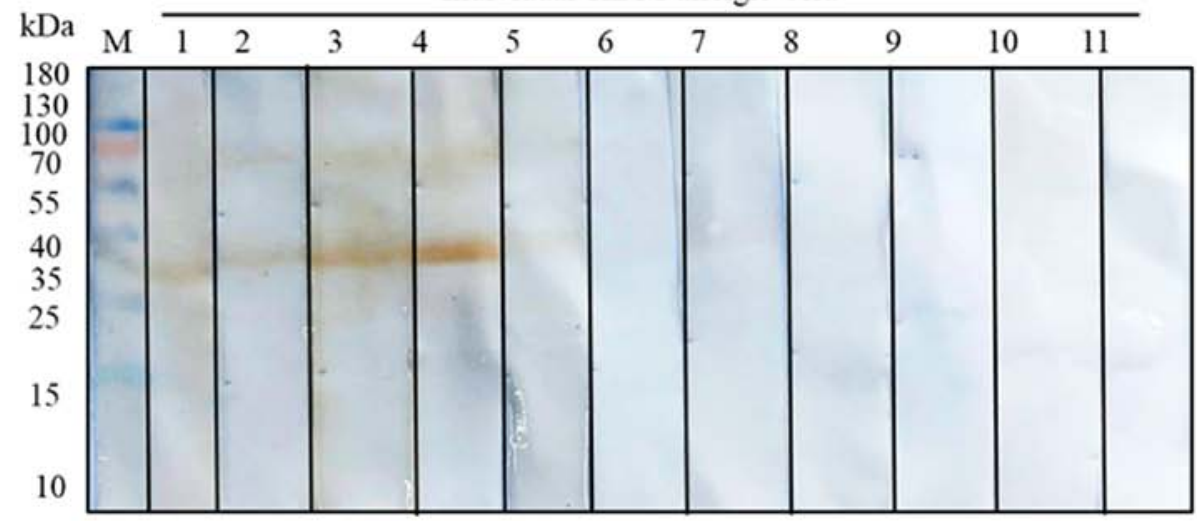

Figure 3. In vitro IgE binding reactivity of $r$ Der $\mathrm{f} 23$. (A) sIgE binding reactivity to $\mathrm{rDer} \mathrm{f} 23$ revealed by IgE ELISA. Dot blot analysis of $\mathrm{rDer} \mathrm{f} 23 \mathrm{in}$ (B) HDM allergic sera from individual patients and (C) sera from non-allergic individuals. Der $\mathrm{f} 1 / 2$ was used as the positive control. IgE western blot analysis of $\mathrm{rDer} \mathrm{f} 23$ with (D) HDM allergic sera from individual patients and (E) sera from non-allergic individuals (E). ${ }^{* *} \mathrm{P}<0.05$. rDer $\mathrm{f} 23$, recombinant Dermatophagoides farina 23 protein; rDer f1/2, recombinant Der f 1-Der f 2 fusion protein; HDM, house dust mite; sIgE, specific immunoglobulin E; OD, optical density.

allergens (34). The IgE ELISA experiment conducted in the present study to examine the role of the $\mathrm{P} 2$ region of Der f 23, which is not present in Der p 23, in the allergenicity of Der f 23 indicated that the rDer f 23 and DsbA-Der f 23 $\triangle \mathrm{P} 2$ proteins exhibited positive sIgE binding activity with HDM allergic sera, whereas DsbA-P2 did not. In addition, 
A
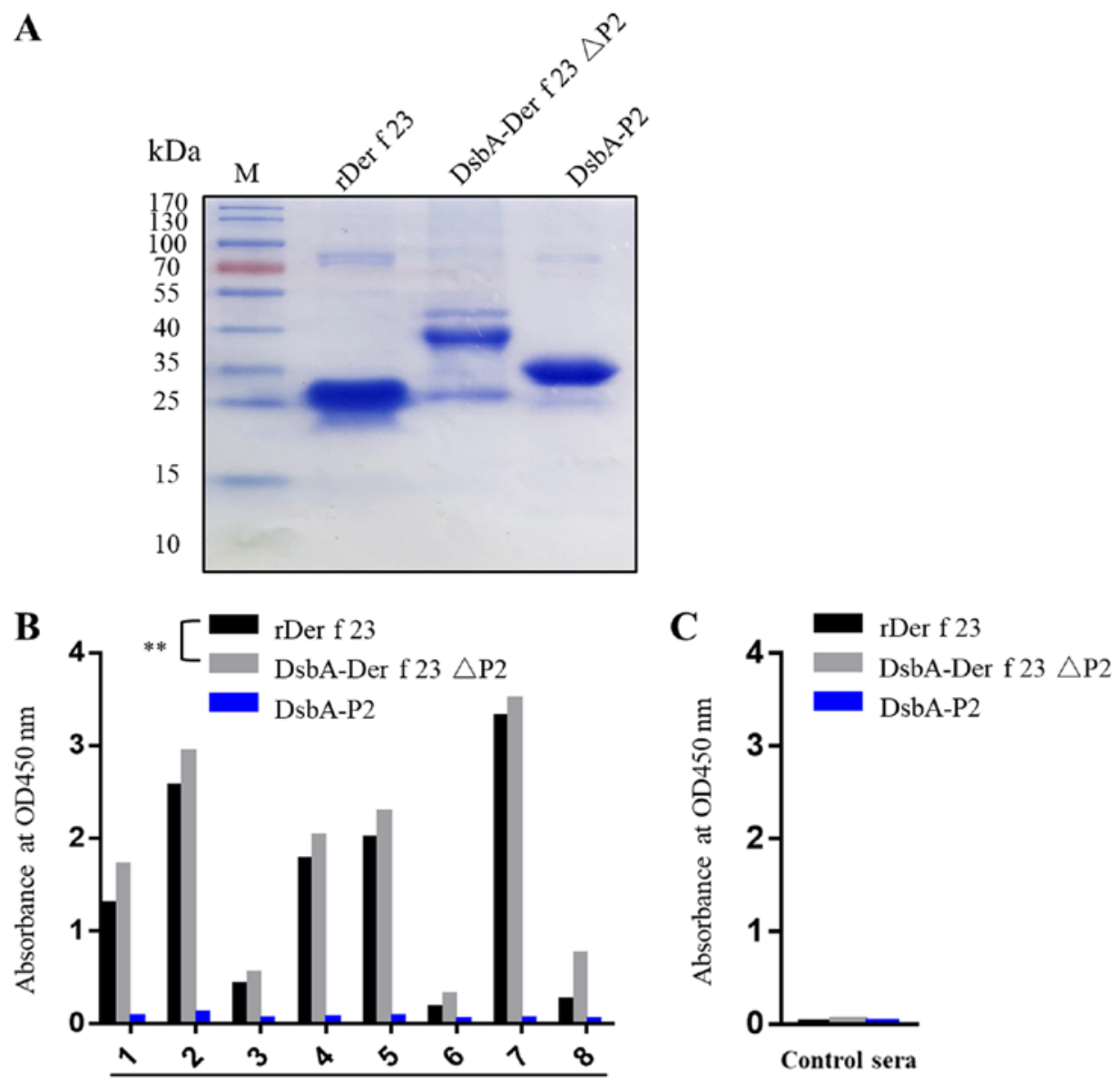

Individual HDM allergic sera

D $\quad$ Individual HDM allergic sera

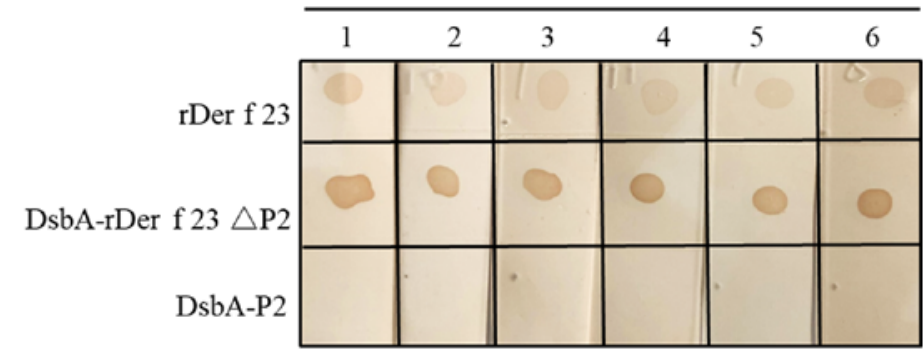

$\mathbf{E}$

Individual non-allergic sera

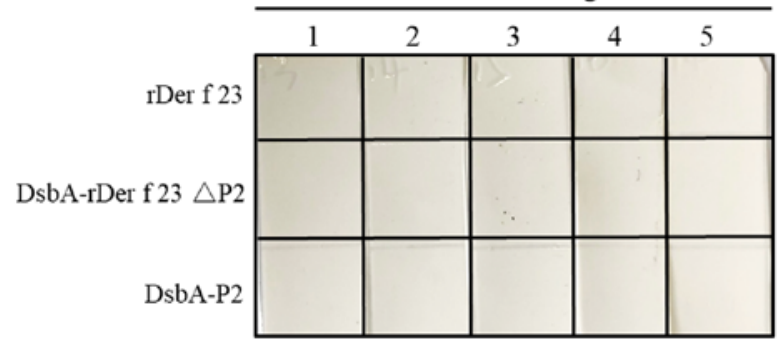

Figure 4. Effect of $\mathrm{P} 2$ region of Der $\mathrm{f} 23$ on sIgE binding ability. The rDer f 23, DsbA-rDer f 23 $\Delta \mathrm{P} 2$, and DsbA-P2 was obtained using an $E$. coli system and subjected to IgE binding assays. (A) SDS-PAGE of purified DsbA-rDer f $23 \Delta \mathrm{P} 2$ and DsbA-P2. IgE ELISA of rDer f 23, DsbA-rDer $\mathrm{f} 23 \Delta \mathrm{P} 2$, and DsbA-P2 in (B) HDM allergic sera from 8 patients and (C) mixed sera from 5 non-allergic controls. sIgE binding dot blot assay of (D) HDM allergic sera from individual patients and (E) sera from non-allergic controls. ${ }^{* *} \mathrm{P}<0.05$. P2, Der f $23 \Delta \mathrm{P} 2$ protein; rDer f 23, recombinant Dermatophagoides farinae 23 protein; sIgE, specific immunoglobulin E; HDM, house dust mite; OD, optical density.

the optical density value at $450 \mathrm{~nm}$ for DsbA-Der f $23 \Delta \mathrm{P} 2$ reacting with SIgE from HDM allergic sera was increased compared with that of rDer $\mathrm{f} 23$. Furthermore, the IgE dot blot experiment revealed a more marked reaction for DsbA-Der $\mathrm{f}$ $23 \Delta \mathrm{P} 2$ compared with rDer f 23 in HDM allergic sera from 6 individuals, consistent with the results of the western blot 
analysis. We hypothesized that the $\mathrm{P} 2$ domain region affects the spatial location of the IgE binding epitope, but this requires additional studies to confirm. Taken together, these results indicate that the addition of the $\mathrm{P} 2$ domain has the ability to affect the allergenic capabilities of HDM allergens.

In conclusion, Der $\mathrm{f} 23$ was identified and characterized using sIgE binding assays in vitro, and Der f 23 immunogenicity was confirmed with in vivo SPTs. Based on these data, Der f 23 was included in the WHO/IUIS allergen database (21). The results of the present study suggested that Der $\mathrm{f} 23$ is an important allergen of $D$. farinae that interacts with sIgEs by way of conformational epitopes. An amino acid sequence difference between Der f 23 and Der p 23, namely the $\mathrm{P} 2$ region that is present only in the former, was demonstrated to affect the sIgE binding activity of Group 23 HDM allergens. These results contribute to the theoretical basis for the diagnosis and treatment of HDM allergies.

\section{Acknowledgements}

Not applicable.

\section{Funding}

The present study was supported in part by research funding from the National Natural Science Foundation of China (grant no. 81571570), and Guangdong Province (grant nos. 2018A050506083, 2014A030313563, 2016A030313039 and 2017A010105014) and Shenzhen City (grant no. JCYJ20150626141652681 and 2016 Biochemistry Discipline Construction).

\section{Availability of data and materials}

All data generated or analyzed in the present study are available from the corresponding author upon reasonable request.

\section{Authors' contributions}

YH performed the experiments and wrote the draft manuscript. CD, YS, JialC and ZZhan participated in the experiments. JiajC and $\mathrm{KJ}$ designed the study and wrote the manuscript. ZZhao made revisions to the manuscript and participated in study design. All of the authors reviewed and approved the final version of the manuscript.

\section{Ethics approval and consent to participate}

Ethics approval was obtained from the First Affiliated Hospital of Guangzhou Medical College (approval no. 2012-51). All procedures involving human participants were in accordance with the ethical standards of the committee of The First Affiliated Hospital of Guangzhou Medical College. All participants voluntarily agreed to participate, and all provided written informed consent.

\section{Patient consent for publication}

All participants voluntarily agreed to participate, and all provided written informed consent.

\section{Competing interests}

The authors declare that they have no competing interests.

\section{References}

1. Iversen M, Korsgaard J, Hallas T and Dahl RJ: Mite allergy and exposure to storage mites and house dust mites in farmers. Clin Exp Allergy 20: 211-219, 1990.

2. Custovic A, Taggart SC and Woodcock A: House dust mite and cat allergen in different indoor environments. Clin Exp Allergy 24: 1164-1168, 1994.

3. Krantz GW: A manual of acarology (Oregon State University Book Stores) pp 194-195, 2010.

4. Kim HK, Yun YK and Ahn YJ: Fumigant toxicity of cassia bark and cassia and cinnamon oil compounds to Dermatophagoides farinae and Dermatophagoides pteronyssinus (Acari: Pyroglyphidae). Exp Appl Acarol 44: 1-9, 2008.

5. Arshad SH, Bateman B, Sadeghnejad A, Gant C and Matthews SM: Prevention of allergic disease during childhood by allergen avoidance: The Isle of Wight prevention study. J Allergy Clin Immunol 119: 307-313, 2007.

6. Suh J, Vaccaro L and Bogart R: Methods for controlling dust mites and the allergens produced by dust mites. (US), 2002.

7. Lau S, Illi S, Sommerfeld C, Niggemann B, Bergmann R, von Mutius E and Wahn U: Early exposure to house-dust mite and cat allergens and development of childhood asthma: A cohort study. Multicentre Allergy Study Group. Lancet 356: 1392-1397, 2000.

8. Gelber LE, Seltzer LH, Bouzoukis JK, Pollart SM, Chapman MD and Platts-Mills TA: Sensitization and exposure to indoor allergens as risk factors for asthma among patients presenting to hospital. Am Rev Respir Dis 147: 573-578, 1993.

9. Kim HS, Kang SH, Won S, Lee EK, Chun YH, Yoon JS, Kim HH and Kim JT: Immunoglobulin E to allergen components of house dust mite in Korean children with allergic disease. Asia Pac Allergy 5: 156-162, 2015.

10. Charpin D, Birnbaum J, Haddi E, Genard G, Lanteaume A, Toumi M, Faraj F, Van der Brempt X and Vervloet D: Altitude and allergy to house-dust mites. A paradigm of the influence of environmental exposure on allergic sensitization. Am Rev Respir Dis 143: 983-986, 1991.

11. Pittner G, Vrtala S, Thomas WR, Weghofer M, Kundi M, Horak F, Kraft D and Valenta R: Component-resolved diagnosis of house-dust mite allergy with purified natural and recombinant mite allergens. Clin Exp Allergy 34: 597-603, 2010.

12. Custovic A, Taggart SC, Francis HC, Chapman MD and Woodcock A: Exposure to house dust mite allergens and the clinical activity of asthma. J Allergy Clin Immunol 98: 64-72, 1996.

13. Boyce JA, Assa'a A, Burks AW, Jones SM, Sampson HA, Wood RA, Plaut M, Cooper SF, Fenton MJ, Arshad SH, et al: Guidelines for the diagnosis and management of food allergy in the United States: Summary of the NIAID-sponsored expert panel report. Nutrition 27: 253-267, 2011.

14. Novakova SM, Staevska MT, Novakova PI, Yoncheva MD, Bratoycheva MS, Musurlieva NM, Tzekov VD and Nicolov DG: Quality of life improvement after a three-year course of sublingual immunotherapy in patients with house dust mite and grass pollen induced allergic rhinitis: Results from real-life. Health Qual Life Outcomes 15: 189, 2017.

15. Chapman MD, Smith AM, Vailes LD, Arruda LK, Dhanaraj V and Pomés A: Recombinant allergens for diagnosis and therapy of allergic diseases. J Allergy Clin Immunol 106: 409-418, 2000.

16. Sub-Committee WIAN: WHO/IUIS Allergen Tree View. (http:// www.allergen.org/treeview.php). Accessed May 23, 2019.

17. Chapman MD and Platts-Mills TA: Purification and characterization of the major allergen from Dermatophagoides pteronyssinus-antigen P1. J Immunol 125: 587-592, 1980.

18. Maeda S, Maeda S, Shibata S, Chimura N and Fukata T: House dust mite major allergen Der f 1 enhances proinflammatory cytokine and chemokine gene expression in a cell line of canine epidermal keratinocytes. Vet Immunol Immunopathol 131: 298-302, 2009.

19. Park GM, Lee SM, Lee IY, Ree HI, Kim KS, Hong CS and Yong TS: Localization of a major allergen, Der $\mathrm{p} 2$, in the gut and faecal pellets of Dermatophagoides pteronyssinus. Clin Exp Allergy 30: 1293-1297, 2010. 
20. Ichikawa S, Hatanaka H, Yuuki T, Iwamoto N, Kojima S, Nishiyama C, Ogura K, Okumura Y and Inagaki F: Solution structure of Der $\mathrm{f} 2$, the major mite allergen for atopic diseases. J Biol Chem 273: 356-360, 1998.

21. Weghofer M, Grote M, Resch Y, Casset A, Kneidinger M, Kopec J, Thomas WR, Fernández-Caldas E, Kabesch M, Ferrara R, et al: Identification of Der $\mathrm{p} 23$, a peritrophin-like protein, as a new major Dermatophagoides pteronyssinus allergen associated with the peritrophic matrix of mite fecal pellets. J Immunol 190: 3059-3067, 2013.

22. Mueller GA, Randall TA, Glesner J, Pedersen LC, Perera L, Edwards LL, DeRose EF, Chapman MD, London RE and Pomés A: Serological, genomic, and structural analyses of the major mite allergen Der p 23. Clin Exp Allergy 46: 365-376, 2016.

23. Chan TF, Ji KM, Yim AK, Liu XY, Zhou JW, Li RQ, Yang KY, Li J, Li M, Law PT, et al: The draft genome, transcriptome, and microbiome of Dermatophagoides farinae reveal a broad spectrum of dust mite allergens. J Allergy Clin Immunol 135: 539-548, 2015.

24. Banerjee S, Weber M, Blatt K, Swoboda I, Focke-Tejkl M, Valent P, Valenta R and Vrtala S: Conversion of Der $\mathrm{p} 23$, a new major house dust mite allergen, into a hypoallergenic vaccine. J Immunol 192: 4867-4875, 2014.

25. Tovey ER, Chapman MD and Platts-Mills TA: Mite faeces are a major source of house dust allergens. Nature 289: 592-593, 1981

26. Fanuel S, Tabesh S, Sadroddiny E and Kardar GA: Analysis of predicted B and T-cell epitopes in Der p 23, allergen from Dermatophagoides pteronyssinus. Bioinformation 13: 307-312, 2017.

27. Randall TA, Mullikin JC and Mueller GA: The Draft Genome Assembly of Dermatophagoides pteronyssinus supports identification of novel allergen isoforms in Dermatophagoides species. Int Arch Allergy Immunol 175: 136-146, 2018.
28. Gieras A, Cejka P, Blatt K, Focke-Tejk1 M, Linhart B, Flicker S, Stoecklinger A, Marth K, Drescher A, Thalhamer J, et al: Mapping of conformational IgE epitopes with peptide-specific monoclonal antibodies reveals simultaneous binding of different IgE antibodies to a surface patch on the major birch pollen allergen, Bet v 1. J Immunol 186: 5333-5344, 2011.

29. Holdom MD, Davies AM, Nettleship JE, Bagby SC, Dhaliwal B, Girardi E, Hunt J, Gould HJ, Beavil AJ, McDonnell JM, et al: Conformational changes in $\mathrm{IgE}$ contribute to its uniquely slow dissociation rate from receptor FceRI. Nat Struct Mol Biol 18: 571-576, 2011.

30. Laver WG, Air GM, Webster RG and Smith-Gill SJ: Epitopes on protein antigens: Misconceptions and realities. Cell 61: 553-556, 1990

31. Sela M: Antigenicity: Some molecular aspects. Science 166: 1365-1374, 1969.

32. Crameri RJ: Correlating IgE reactivity with three-dimensional structure. Biochem J 376: e1-e2, 2003.

33. Szalai K, Fuhrmann J, Pavkov T, Scheidl M, Wallmann J, Brämswig KH, Vrtala S, Scheiner O, Keller W, Saint-Remy JM, Neumann D, et al: Mimotopes identify conformational B-cell epitopes on the two major house dust mite allergens Der p 1 and Der p 2. Mol Immunol 45: 1308-1317, 2008.

34. Chruszcz M, Chapman MD, Vailes LD, Stura EA, Saint-Remy JM, Minor W and Pomés A: Crystal structures of mite allergens Der $\mathrm{f} 1$ and Der $\mathrm{p} 1$ reveal differences in surface-exposed residues that may influence antibody binding. J Mol Biol 386: 520-530, 2009.

This work is licensed under a Creative Commons Attribution-NonCommercial-NoDerivatives 4.0 International (CC BY-NC-ND 4.0) License. 